\title{
Intoxicação plumbífera aguda no tecido cardíaco em ratos Wistar
}

\author{
Effects of acute lead poisoning on cardiac tissue of Wistar rats
}

Matheus Folgearini Silveira ${ }^{[a]}$, Sueli Pércio Quináia ${ }^{[b]}$,Jayme Augusto Peres ${ }^{[c]}$

[a] Médico-veterinário, mestre em Sanidade Animal, professor do Departamento de Medicina Veterinária da Universidade Estadual do Centro-Oeste (Unicentro), Guarapuava, PR - Brasil, e-mail: matheusmedvet@gmail.com

[b] Doutora em Química, professora da Universidade Estadual do Centro-Oeste (Unicentro), Guarapuava, PR - Brasil, e-mail: spquinaia@gmail.com

[c] Médico-veterinário, doutor em Toxicologia Ambiental, professor da Universidade Estadual do Centro-Oeste (Unicentro), Guarapuava, PR - Brasil, e-mail: perespatologia@hotmail.com

\section{Resumo}

A insuficiência cardíaca congestiva é uma síndrome clínica caracterizada por disfunção cardíaca com hemodinâmica anormal, liberação de citocinas, ativação neuroendócrina e retenção renal de sódio e água. Diversas etiologias são associadas; porém, a importância da intoxicação por metais pesados na musculatura cardíaca e seu sistema de condução têm sido pouco estudados. Neste estudo, objetivou-se a caracterização histomorfológica do coração de modelos biológicos submetidos à intoxicação aguda por chumbo. Dezoito ratos Wistar foram divididos em três grupos, sendo o chumbo (G1) testado em conjunto com o cobre (G2) e controle. Os animais submetidos à intoxicação experimental por chumbo apresentaram lesões em segmentos miocárdicos em parede livre do ventrículo esquerdo com infiltrado inflamatório mononuclear associado com a degeneração de miofibrilas. Observou-se alteração em gânglios nervosos cardíacos com degeneração neuronal multifocal, localizados principalmente no ventrículo esquerdo e no septo interventricular. Nos demais grupos não se identificaram lesões referentes ao tecido muscular cardíaco e/ou de condução. Concluiu-se que o chumbo possui características de alteração estrutural cardíaca importante, especialmente no que diz respeito aos danos no sistema nervoso cardíaco, devendo-se incluir no rol de possibilidades de eventuais disritmias em animais domésticos.

Palavras-chave: Chumbo. Coração. Toxicologia. Cardiologia.

\section{Abstract}

Congestive heart failure is a clinical syndrome characterized by cardiac dysfunction with abnormal hemodynamics, cytokine release, neuroendocrine activation and sodium and water retention. Many etiologies are associated with heavy metal poisoning, but its importance on cardiac muscle and conduction system have been 
scarcely studied. This study aimed to characterize the cardiac histopathological findings on a biological model submitted to acute lead poisoning. Eighteen male Wistar rats were distributed among three groups, comprising lead treatment (G1), copper treatment (G2) and control. The animals experimentally intoxicated with lead presented lesions in the free wall myocardial segments of the left ventricle, with mononuclear inflammatory infiltrate associated to myofibril degeneration. It was also observed alterations on cardiac nervous ganglia with multifocal neuronal degeneration localized mainly in the left ventricle and interventricular septum. The other two groups did not present any relevant lesions in the cardiac muscle and/or its conduction system. Based on these findings, lead causes important structural cardiac tissue alterations, especially in the cardiac nervous conduction system. Then, poisoning with this element should be included among the possible causes of congestive heart failure in domestic animals.

Keywords: Lead. Heart. Toxicology. Cardiology.

\section{Introdução}

A cardiologia é um ramo da Medicina Veterinária que evoluiu nos últimos anos a partir do desenvolvimento e utilização de métodos de diagnóstico auxiliares. Contribuíram para esse processo também a conscientização mundial da importância das doenças cardiovasculares em humanos, incluindo-se a doença de Chagas (PASCON et al., 2010), e o emprego de animais de companhia como modelo biológico eficaz em pesquisas com cardiomiopatias tóxicas, estudos hemodinâmicos, alterações vasculares e uso de biomarcadores (HASENFUSS, 1998). Muitos mitos têm sido superados, como a não regeneração dos cardiomiócitos (BERGMANN et al., 2009), o que ressalta a importância de pesquisas de base contínua, assim como ensaios clínicos na cardiologia.

Dentre as cardiopatias, a insuficiência cardíaca não pode ser considerada apenas um distúrbio contrátil ou uma doença cardíaca isolada. As manifestações clínicas são resultado de mudanças nos componentes e mediadores celulares cardíacos que realizam o controle homeostático (COHN; FERRARI; SHARPE, 2000; SCHWARTZ; MORAIS, 2005).

A insuficiência cardíaca congestiva (ICC) é uma síndrome clínica, na qual a deficiência no débito cardíaco proporciona uma hemodinâmica inadequada, gerando mecanismos compensatórios que incluem a ativação neuro-hormonal do sistema renina angiotensina, citocinas e bradicininas circulantes. Uma lesão cardíaca ou vascular que limita a ejeção cardíaca e reduz a pressão arterial sanguínea (PAS) resulta em insuficiência cardíaca (SCHWARTZ; MORAIS, 2005).
A resposta compensatória à insuficiência cardíaca suporta a PAS, todavia promove concomitantemente um estado de má adaptação que leva a uma morbidade substancial e mortalidade (BONAGURA; LEHMKUHL; MORAIS, 2006; SCHWARTZ; MORAIS, 2005).

A ICC avançada, assim como a terapia desta, geralmente é associada a alterações na função renal e diversas anormalidades hídricas, eletrolíticas e bioquímica sérica. Alguns desses distúrbios são considerados leves a bem tolerados, mas outros como hiponatremia e insuficiência renal aguda indicam uma disfunção circulatória grave e a necessidade de uma terapia urgente (BONAGURA; LEHMKUHL; MORAIS, 2006).

0 remodelamento cardíaco pode ser descrito como uma condição fisiológica e patológica que advém de infarto do miocárdio, aumento de pressão (estenose aórtica, hipertensão), doença inflamatória do músculo cardíaco (miocardite), cardiomiopatia dilatada ou sobrecarga de volume (regurgitação valvar). Apesar das diferentes etiologias, essas doenças partilham eventos moleculares, bioquímicos e mecânicos semelhantes (COHN; FERRARI; SHARPE, 2000).

Dentre as possibilidades descritas, a cardiomiopatia tóxica é subestimada como importante fator deflagrador de alterações hemodinâmicas. Várias toxinas têm influência direta, como a toxina botulínica, a ingestão de plantas tóxicas e os metais pesados. Observa-se, especialmente dentre estes, o chumbo como um dos principais elementos cardiotóxicos (COHN; FERRARI; SHARPE, 2000; FEITOSA FILHO et al., 2011).

As consequências do chumbo no organismo estão associadas à inativação de ATPases de cálcio, 
sódio e potássio, ácido aminolevulínico desidratase (ALAD), ácido 5-aminolevulínico (ALA) e, principalmente, às enzimas relacionadas à detoxicação hepática, como a citocromo $\mathrm{P}_{450}$. Em adição, o chumbo desencadeia um processo degenerativo dos sistemas nervoso central e periférico, desmielinização e degenerações axonais (ROCHA; ROSA; CARDOSO, 2004; SPINOSA; GÓRNIAK; PALERMO-NETO, 2008).

Com base nessas considerações, o presente trabalho teve o objetivo de analisar os efeitos da intoxicação plumbífera no tecido cardíaco, assim como detectar os tipos de alteração histopatológica correlacionada com as lesões no sistema cardiovascular.

\section{Materiais e métodos}

0 trabalho foi desenvolvido experimentalmente com a utilização de 18 ratos da linhagem Wistar, machos, com 120 dias, pesando aproximadamente 250 gramas. Os animais foram separados em seis caixas contendo três animais cada, e receberam água e ração apropriada ad libitum, sendo o experimento conduzido em sala com temperatura e ventilação adequada no Laboratório de Anatomia Patológica Veterinária da Universidade Estadual do Centro-Oeste (Unicentro).

O período de uma semana foi utilizado para a ambientação dos animais e o processo experimental iniciado foi mantido por sete dias consecutivos. Os animais foram manipulados durante o procedimento sempre pelas mesmas pessoas. 0 presente projeto foi aprovado pelo Comitê de Ética em Uso de Animais (CEUA) da Universidade Estadual do Centro-Oeste (Unicentro) mediante ofício 024/2012 e protocolado sob o número 032/2011, seguindo as prerrogativas éticas estabelecidas.

\section{Segmentação dos grupos}

Foram selecionados três elementos: água $\left(\mathrm{H}_{2} \mathrm{O}\right)$, para o grupo controle; chumbo $(\mathrm{Pb})$ no formato nitrato de chumbo $\mathrm{Pb}\left(\mathrm{NO}_{3}\right)_{2}$, que foi o elemento de interesse; e cobre $(\mathrm{Cu})$ na forma de sulfato de cobre $\left(\mathrm{Cu}_{2} \mathrm{SO}_{4}\right)$ como elemento comparativo, servindo de controle de qualidade do chumbo. Os metais foram utilizados na forma de sais por causa de sua não diluição em meio líquido na sua forma pura. Para cada elemento, foram separados dois grupos: GI$\mathrm{H}_{2} \mathrm{O}$ e $\mathrm{GII}-\mathrm{H}_{2} \mathrm{O}$; GI-Cu e GII-Cu; GI-Pb e GII-Pb.

\section{Preparo das amostras}

Para preparo das amostras, foram dissolvidos os sais de $\mathrm{Pb}\left(\mathrm{NO}_{3}\right)_{2}$ e $\mathrm{Cu}_{2} \mathrm{SO}_{4}$ em uma proporção de $10 \mathrm{~g}$ para $100 \mathrm{~mL}$ de água. As soluções preparadas foram aplicadas para cada grupo correspondente aos elementos de modo que, para cada quilo de animal, $5 \mathrm{~mL}$ da solução foram inoculados pela técnica de gavagem, e todos os dias antes da inoculação dos elementos foi elaborada uma nova solução.

0 experimento teve duração de sete dias e todos os animais foram pesados diariamente para a obtenção da média necessária de fornecimento dos elementos. Os animais também foram submetidos a restrição dietética e hídrica por aproximadamente 15 min antes da técnica de gavagem, com a finalidade de favorecer a absorção do elemento inoculado.

\section{Eutanásia e exame necroscópico}

No oitavo dia de condução do experimento, os animais foram eutanasiados no Laboratório Didático do Departamento de Medicina Veterinária em câmara de gás carbônico $\left(\mathrm{CO}_{2}\right)$ e observados até que suas funções vitais tivessem parado totalmente. Em seguida, todos foram conduzidos ao procedimento de necropsia, realizada no Laboratório de Anatomia Patológica Veterinária, para avaliação e descrição das alterações macroscópicas e coleta das amostras para processamento histopatológico e toxicológico.

\section{Histopatologia}

Para o processamento histopatológico foram retirados os seguintes órgãos: pele, pulmão, coração, fígado, baço, intestino, estômago, fêmur, dentes, testículo, epidídimo, músculo estriado esquelético do membro pélvico, encéfalo e cerebelo. As amostras foram coletadas em frascos plásticos 
devidamente identificados, fixadas em formalina tamponada a $10 \%$ e processadas pela técnica histológica e coloração padrão Hematoxilina e Eosina (H\&E).

\section{Exame toxicológico}

Foram selecionados fígado e rins, devido ao seu papel na detoxicação e excreção; esses órgãos foram acondicionados em sacos plásticos devidamente identificados e refrigerados à temperatura de $4{ }^{\circ} \mathrm{C}$. 0 processo de mineralização do material foi realizado nas instalações do Laboratório de Química Analítica do Departamento de Química da Unicentro. 0 recipiente para análise das amostras, denominado "vaso de reação de polietileno" (Hostaflon), foi descontaminado com uma solução de ácido nítrico a 10\%, e posteriormente procedeu-se à lavagem com água deionizada por três vezes (tríplice lavagem). 0 processo constituiu em coletar aproximadamente $6 \mathrm{~g}$ do material, utilizando de $8 \mathrm{~mL}$ de ácido nítrico $\left(\mathrm{HNO}_{3}\right)$ para a digestão. As amostras foram levadas ao forno de micro-ondas (PROVECTO DGT 100 Plus), onde foi utilizada a programação específica conforme o tipo de amostra a ser mineralizada.

Após atingirem a temperatura ambiente, os vasos de reação foram conduzidos até à câmara de exaustão (Capela), onde foram abertos; as amostras foram colocadas em frascos de polietileno com tampa rosqueável (Starsted) e devidamente identificados. Em seguida, foi avolumado o frasco da amostra para $25 \mathrm{~mL}$ e obtido uma solução translúcida da solução mineralizada. A análise foi realizada no aparelho de espectrofotometria de absorção atômica em chamas (FAAS - Atomic Absorption Spectrometry In Flame) (GBC AA 932). Todo procedimento foi realizado com a utilização de equipamentos de proteção individual, conforme a recomendação do manual de biossegurança (TEIXEIRA; VALLE, 2002).

\section{Análise estatística}

A análise estatística aplicada foi o Teste de Tukey, sendo considerado significativo $\mathrm{p}<0,05$. Contudo, dada a natureza multiparamétrica dos dados, foi realizado o Teste de Spearman por meio do software STATISTIX ${ }^{\circledR}$.

\section{Resultados e discussão}

A análise pela FAAS comprovou que os animais foram intoxicados pelos elementos chumbo e cobre, considerando que o ambiente e a metodologia utilizada para o desenvolvimento do projeto foi livre de contaminação desses metais pesados.

Os níveis de chumbo detectáveis foram de $4,14 \mu \mathrm{g} / \mathrm{g}$ no fígado e $86,86 \mu \mathrm{g} / \mathrm{g}$ nos rins, o que demonstrou o caráter de acumulação desse elemento no fígado e a sua excreção renal. Ao analisar esses órgãos, notou-se, no fígado, necrose multifocal associada à reação inflamatória mononuclear perivascular com tumefação de células de Küpfer. Nos rins, evidenciou-se degeneração hidrópica de células tubulares associada à picnose e condensação cromatínica (FEITOSA FILHO et al., 2011; McGAVIN; ZACHARY, 2009).

Nesse experimento, o chumbo causou, quando em níveis tóxicos, taquipneia e alterações de musculatura lisa e estriada também descritas em outras localidades. Essas alterações são motivadas pelo fato de o chumbo competir com receptores de cálcio, que estimulam movimentação de fibras musculares (MOREAU; SIQUEIRA, 2008; OGA CAMARGO; BATISTUZZO, 2008).

Os animais submetidos à intoxicação experimental por esse elemento apresentaram lesões em segmentos miocárdicos em parede livre do ventrículo esquerdo, com infiltrado inflamatório mononuclear associado com degeneração de miofibrilas (Figura 1). Além disso, observou-se alteração em gânglios nervosos cardíacos com degeneração neuronal multifocal, localizados principalmente em ventrículo esquerdo e septo interventricular. Nos demais grupos, não se identificaram lesões dignas de nota referente ao tecido muscular cardíaco e/ou de condução. Dadas tais alterações cardiorrespiratórias, a sintomatologia de comprometimento hemodinâmico condiz com a morfologia histopatológica observada nos animais intoxicados por chumbo e ressalta, principalmente, o estresse oxidativo (FEITOSA FILHO et al., 2011; McGAVIN; ZACHARY, 2009; SCHWARTZ; MORAIS, 2005).

Cabe salientar que o córtex e o cerebelo apresentaram-se extremamente sensíveis ao elemento 


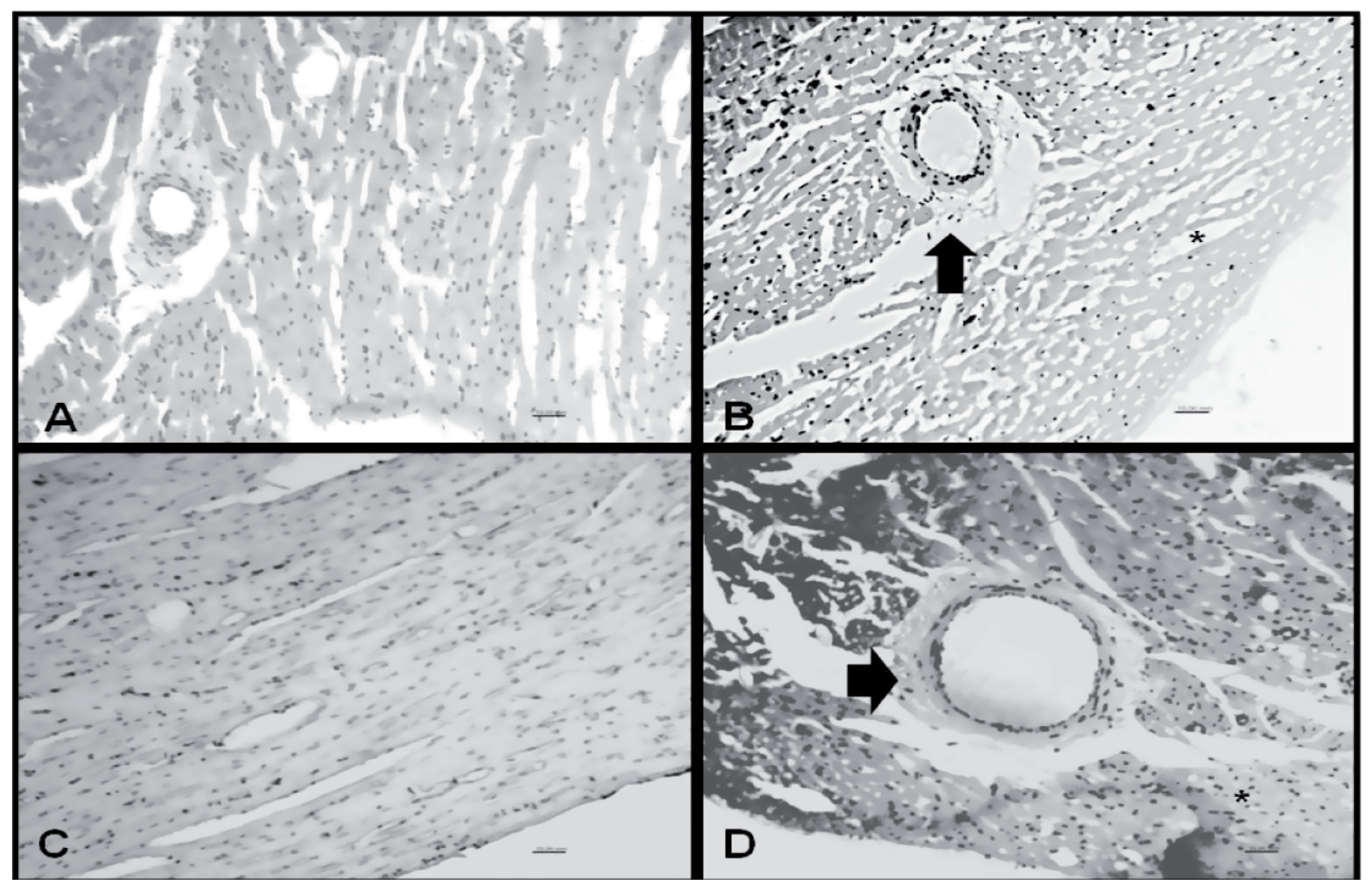

Figura 1 - Achados histopatológicos do chumbo (B, D) em relação ao grupo controle (A, C)

Fonte: Dados da pesquisa.

Nota: Em B, evidencia-se a degeneração de miofibrilas cardíacas (asterisco) e vasculite (seta) com necrose acentuada multifocal coalescente. Em D, 0 arranjo dos filamentos denota intensa necrose e espessamento da túnica adventícia (seta). Barra: $10 \mathrm{~mm}$.

chumbo, que causou processos degenerativos difusos e desmielinização. Esses processos podem ter efeitos acumulativos e/ou tardios em razão de as alterações serem progressivas, difusas e irreversíveis (McGAVIN; ZACHARY, 2009). Considerando os achados no sistema nervoso central, esses podem ser correlacionados com os danos no tecido de condução cardíaco, o que pode conduzir a eventuais disritmias e cardiomiopatias consideráveis, insuficiência cardíaca, arritmias e morte súbita (COHN; FERRARI; SHARPE, 2000; FEITOSA FILHO et al., 2011; SCHWARTZ; MORAIS, 2009).

\section{Conclusão}

O chumbo é capaz de induzir alterações estruturais cardíacas importantes no modelo biológico empregado, especialmente no que tange ao sistema de condução cardíaco. Portanto, esse elemento deve ser incluído no rol de possibilidades de eventuais disritmias em animais domésticos.

\section{Agradecimentos}

Ao Prof. Dr. Adriano Torres de Oliveira Carrasco, pela obtenção das imagens histopatológicas deste trabalho.

\section{Referências}

BERGMANN, O. et al. Evidence for cardiomyocyte renewal in humans. Science, v. 324, n. 98, 2009. PMid:19342590.

BONAGURA, J. D.; LEHMKUHL, L. B.; MORAIS, H. A. Fluid and diuretic therapy in heart failure. In: DI BARTOLA, S. P. (Ed.). Fluid, electrolyte and acid base disorders in small animal practice. 3th. ed. Saint Louis: Saunders, 2006. p. 490-518. 
COHN, J. N.; FERRARI, R.; SHARPE, N. Cardiac remodeling - concepts and clinical implications: a consensus paper from an international forum on cardiac remodeling. Journal of the American College of Cardiology, v. 35, n. 3, p. 569-582, 2000. doi:10.1016/ S0735-1097(99)00630-0.

FEITOSA FILHO, F. H. et al. Cardiomiopatia arritmogênica do ventrículo direito. Revista da Sociedade Brasileira de Clínica Médica, v. 9, n. 1, p. 67-73, 2011.

HASENFUSS, G. Animal models of human cardiovascular disease, heart failure and hypertrophy. Cardiovascular Research, v. 39, p. 60-76, 1998. doi:10.1016/ S0008-6363(98)00110-2.

McGAVIN, M. D.; ZACHARY, J. F. Bases da patologia em veterinária. 4. ed. Rio de Janeiro: Elsevier, 2009.

MOREAU, R. L. M.; SIQUEIRA, M. E. P. B. Toxicologia analítica. Rio de Janeiro: Guanabara Koogan, 2008.

OGA, S.; CAMARGO, M. M. A.; BATISTUZZO, J. A. 0. Fundamentos de toxicologia. 3. ed. Atheneu Editora: São Paulo, 2008.

PASCON, J. P. E. et al. Clinical characterization of chronic chagasic cardiomyopathy in dogs. Pesquisa Veterinária Brasileira, v. 30, n. 2, p. 115-120, 2010. doi:10.1590/ S0100-736X2010000200003.
ROCHA, J. C.; ROSA, A. H.; CARDOSO, A. A. Introdução à química ambiental. Porto Alegre: Bookman, 2004.

SCHWARTZ, D. S.; MORAIS, H. A. de. Pathophysiology of heart failure. In: ETTINGER, S. J.; FELDMAN, E. C. Textbook of veterinary internal medicine: diseases of the dog and cat. 6th. ed. Philadelphia: W. B. Saunders, 2005. p. 914-940. PMid:15965031.

SPINOSA, H. S.; GÓRNIAK, S. L.; PALERMO-NETO, J. Toxicologia aplicada à medicina veterinária. São Paulo: Editora Manole, 2008.

TEIXEIRA, P.; VALLE, S. Mapa de risco e segurança química em biotecnologia. In: TEIXEIRA, P.; VALLE, S. Biossegurança - uma abordagem multidisciplinar. Editora Fiocruz: Rio de Janeiro, 2002. p. 111-131.

Recebido: 16/09/2012 Received: 09/16/2012

Aprovado: 21/12/2012

Approved: $12 / 21 / 2012$ 